\title{
Gambogic acid inhibits growth, induces apoptosis, and overcomes drug resistance in human colorectal cancer cells
}

\author{
CHUANGYU WEN ${ }^{1-3}$, LANLAN HUANG $^{1-3}$, JUNXIONG CHEN $^{1-3}$, MENGMENG LIN $^{1-3}$, \\ WEN LI ${ }^{4}$, BIYAN LU ${ }^{5}$, ZINA JEYAPALAN RUTNAM ${ }^{6}$, AIKICHI IWAMOTO ${ }^{7,8}$, \\ ZHONGYANG WANG ${ }^{1}$, XIANGLING YANG ${ }^{1-3^{*}}$ and HUANLIANG LIU ${ }^{1-3^{*}}$
}

\begin{abstract}
${ }^{1}$ Guangdong Institute of Gastroenterology and the Sixth Affiliated Hospital, Sun Yat-sen University, Guangzhou, Guangdong 510655; ${ }^{2}$ Guangdong Provincial Key Laboratory of Colorectal and Pelvic Floor Diseases, Sun Yat-sen University, Guangzhou, Guangdong 510655 ; $^{3}$ Institute of Human Virology and Key Laboratory of Tropical Disease Control of Ministry of Education, Sun Yat-sen University, Guangzhou, Guangdong 510080; ${ }^{4}$ Guangdong Provincial Key Laboratory of Allergy and Immunology, Guangzhou Medical University, Guangzhou, Guangdong 510260; ${ }^{5}$ Dongguan Health School, Dongguan, Guangdong 523186, P.R. China; ${ }^{6}$ Tumor Vaccine Group, Center for Translational Medicine in Women's Health, University of Washington, Seattle, WA 98195-8050, USA;

${ }^{7}$ Advanced Clinical Research Center, Institute of Medical Science, University of Tokyo, Tokyo 108-8639, Japan
\end{abstract}

Received June 25, 2015; Accepted August 3, 2015

DOI: $10.3892 /$ ijo.2015.3166

\begin{abstract}
The emergence of chemoresistance is a major limitation of colorectal cancer (CRC) therapies and novel biologically based therapies are urgently needed. Natural products represent a novel potential anticancer therapy. Gambogic acid (GA), a small molecule derived from Garcinia hanburyi Hook. f., has been demonstrated to be highly cytotoxic to several types of cancer cells and have low toxicity to the hematopoietic system. However, the potential role of GA in colorectal cancer and its ability to overcome the chemotherapeutic resistance in CRC cells have not been well studied. In the present study, we showed that GA directly inhibited proliferation and induced apoptosis in both 5-fluorouracil (5-FU) sensitive and 5-FU resistant colorectal cancer cells; induced apoptosis via activating JNK signaling pathway. The data, therefore, suggested an alternative strategy to overcome 5-FU resistance in CRC and that GA could be a promising medicinal compound for colorectal cancer therapy.
\end{abstract}

Correspondence to: Dr Xiangling Yang or Professor Huanliang Liu, Guangdong Institute of Gastroenterology and the Sixth Affiliated Hospital, Sun Yat-sen University, 260 Yuancun Er Heng Road, Guangzhou, Guangdong 510655, P.R. China

E-mail: morningyang100@gmail.com

E-mail: liuhuanl@mail.sysu.edu.cn

Present address: ${ }^{8}$ Japan Agency for Medical Research and Development (AMED), Tokyo, Japan

*Contributed equally

Key words: gambogic acid, colorectal cancer, 5-FU resistance, apoptosis, JNK signaling pathway

\section{Introduction}

Colorectal cancer (CRC) is the second leading cause of cancerrelated death, and 5-fluorouracil (5-FU) is still the main chemotherapeutic agent used in the first-line treatment of this disease. The response rate of 5-FU monotherapy is $15 \%$ and thus modulators are added for higher response rates: combination therapy of 5-FU with irinotecan or oxaliplatin (40\%), and the newly developed combination therapy of 5-FU with bevacizumab and cetuximab (60-70\%) (1). However, resistance to chemotherapy is a major cause of mortality in CRC patients. Therefore, other compounds are needed in order to increase treatment efficacy and overcome the 5-FU resistance. Furthermore, the current therapy options with 5-FU cause severe side-effects. Thus, novel agents are urgently required. Natural products are a rich source of anticancer agents and provide novel and more effective anticancer agents for therapeutic use. Gambogic acid (GA) is a small molecule extracted from the traditional Chinese medicine herb Garcinia hanburyi Hook. f. which has been used for a long time in China. GA has a strong cytotoxic effect on a variety of cancers but has very weak effect on the hematologic system (2-5). Importantly, GA has been approved by the China Food and Drug Administration (CFDA) for phase II clinical trial in solid tumor therapy (6). There have been many research studies published demonstrating the anticancer activity of GA (3,7-10). However, the mechanisms of action for the GA anticancer effects are not fully understood. Therefore, further molecular studies need to be conducted in order to further elucidate the mechanism of GA activity. In the present study, we have established an acquired 5-FU resistant cell line to explore the anticancer effect of GA. We demonstrated that GA directly inhibited proliferation and induced apoptosis in both drug sensitive and drug resistant colorectal cancer cells and induced apoptosis via activating the JNK signaling pathway. Data presented here 
demonstrate that GA activates the JNK signaling pathway and overcomes drug resistance in CRC cells. Thus, it could be a promising medicinal compound for colorectal cancer therapy.

\section{Materials and methods}

Cell culture. Human epithelial colorectal adenocarcinoma HCT-15 cells were purchased from the Culture Collection of Chinese Academy of Science (Shanghai, China). Cells were cultured in Dulbecco's modified Eagle's medium (Gibco Life Technologies, Carlsbad, CA, USA) supplemented with $10 \%$ inactivated fetal bovine serum (Gibco Life Technologies), $100 \mathrm{units} / \mathrm{ml}$ penicillin and $10 \mu \mathrm{g} / \mathrm{ml}$ streptomycin (Gibco Life Technologies) in a humidified atmosphere of $5 \% \mathrm{CO}_{2}$ at $37^{\circ} \mathrm{C}$. The 5-FU resistant cell line (HCT-15R) was established from its parental cell line HCT-15 by stepwise exposure to increasing the concentrations of 5-FU, starting at $1 \mu \mathrm{M}$ and ending at $100 \mu \mathrm{M}$. 5-FU $(1 \mu \mathrm{M})$ was included in the culture medium for HCT-15R to maintain the drug resistance. The cells were maintained in 5-FU free medium at least 2 weeks before the experiments.

Reagents. 5-Fluorouracil (Sigma-Aldrich, St. Louis, MO, USA) was dissolved in dimethyl sulphoxide (DMSO) to a $200 \mathrm{mM}$ solution and stored at $-20^{\circ} \mathrm{C}$. SP600125 (SigmaAldrich) was dissolved in DMSO to a $50 \mathrm{mM}$ solution and stored at $-20^{\circ} \mathrm{C}$. Gambogic acid (Sigma-Aldrich) was dissolved in DMSO to a $10 \mathrm{mM}$ stock solution and stored at $-20^{\circ} \mathrm{C}$. PARP, caspase-3, cleaved-caspase-3, caspase-8, Mcl-1, Bcl-xl, Bcl-2, XIAP, survivin, cytochrome $c$, AIF, cyclin D1, p53, JNK and phospho-JNK at Thr183/Tyr185 were from Cell Signaling Technology (Beverly, MA, USA). Cleaved-caspase-9 was purchased from Abcam (Cambridge, MA, USA). Antibodies against caspase-9, $\beta$-actin and anti-mouse immunoglobulin $\mathrm{G}$ and anti-rabbit immunoglobulin $\mathrm{G}$ horseradish peroxidaseconjugated secondary antibodies were from Proteintech Group (Chicago, IL, USA).

Cell proliferation assay by real-time cell impedance analysis. For real-time cell analysis (RTCA), xCELLigence system (Roche Applied Science, Mannheim, Germany) was used to dynamically monitor cell proliferation rates. The experiments were performed following the standard protocol developed by Roche Applied Science. Briefly, cells were seeded into $100 \mu \mathrm{l}$ of media in an E-Plate. Cell proliferation was monitored at set intervals via measuring electrical impedance across microelectrodes on the bottom of E-Plate. The impedance was expressed as cell index (CI), an arbitrary unit. RTCA software, supplied by the manufacturer, was used to analyze the measurements.

Cell viability assay. The effect of GA on cell viability assay was analyzed by MTS assay (Promega, Madison, WI, USA). Briefly, a total of $5 \times 10^{4} / \mathrm{ml}$ cells in $100 \mu 1$ were treated with GA for $72 \mathrm{~h}$. Four hours before culture termination, $20 \mu \mathrm{l}$ MTS labeling mixture (MTS/PMS) was added to each well. The absorbance density was read on a 96 -well plate reader at wavelength $490 \mathrm{~nm}$.

Cell cycle analysis by flow cytometry. Cells incubated with $1 \mu \mathrm{M}$ GA for the indicated times, were collected, washed and fixed with $75 \%$ cold ethanol at $4{ }^{\circ} \mathrm{C}$ overnight. After $75 \%$ ethanol was moved, cells were washed twice in phosphatebuffered saline (PBS) and labeled with propidium iodide (BD Biosciences, Franklin Lakes, NJ, USA). The cell cycle distribution was analyzed by BD FACSCanto II flow cytometry.

Analysis of cell apoptosis by flow cytometry. Apoptosis was determined by flow cytometry using an Annexin V-FITC/PI dual staining kit (Nanjing KeyGen Biotech Co., Ltd., Nanjing, China). Cells incubated with $2 \mu \mathrm{M} \mathrm{GA}$ for the indicated times, were collected, washed and stained in working solution (500 $\mu \mathrm{l}$ binding buffer with $5 \mu \mathrm{l}$ Annexin V-FITC and $5 \mu \mathrm{l}$ propidium iodide) for $15 \mathrm{~min}$ at room temperature in the dark. Cells were then washed and resuspended with binding buffer. Apoptotic cells were determined by BD FACSCanto II flow cytometry. Annexin V-FITC-positive and Annexin V-FITC-plus-PI positive cells were determined as apoptotic cells.

Mitochondrial membrane potential measurement. Mitochondrial membrane potential was detected using a JC-1 mitochondrial membrane potential assay kit (Nanjing KeyGen Biotech Co., Ltd.), following the manufacturer's protocol. Briefly, after treatment, cells were incubated with JC-1 staining solution at $37^{\circ} \mathrm{C}$ for $20 \mathrm{~min}$ and rinsed twice with incubation buffer provided by the kit. Fluorescence intensity of both mitochondrial JC-1 monomers (Green) and aggregates (Red) were detected using a BD FACSCanto II flow cytometry. JC-1 stains the mitochondria of healthy cells red, and apoptotic cells green.

Western blot analysis. Western blot analysis was performed as previously described (11). Briefly, cells were lysed in lysis buffer containing protease and phosphatase inhibitors (Nanjing KeyGen Biotech Co., Ltd.). Protein concentrations were measured using a Bio-Rad assay kit (Bio-Rad Laboratiries, Hercules, CA, USA). Total cellular proteins were separated by SDS-PAGE and transferred to PVDF membranes followed by probed with a primary antibody overnight at $4^{\circ} \mathrm{C}$. The next day, the membrane was washed and incubated with HRP-conjugated secondary antibody at room temperature for $2 \mathrm{~h}$ followed by ECL detection. After detection of protein bands, the membrane was stripped and re-probed with anti- $\beta$ actin antibody to confirm equal loading of samples.

Preparation of cytoplasmic fractions. GA-treated cells were pelleted by centrifugation and rinsed with PBS. Whole cell lysates were prepared in ice-cold lysis buffer (10 mM Hepes, pH 7.9, $10 \mathrm{mM} \mathrm{KCl}, 0.1 \mathrm{mM}$ EDTA, $0.4 \%$ NP-40 with $1 \mathrm{mM}$ DTT, $0.5 \mathrm{mM}$ PMSF, $1 \mathrm{mM} \mathrm{NaF}$ and $1 \mathrm{mM}$ Complete protease inhibitor mix) by pipetting up and down (without bubbling) $\sim 10$ times. After incubation on ice for $10 \mathrm{~min}$, the lysates were centrifuged at $15,000 \mathrm{x} \mathrm{g}$ for $1 \mathrm{~min}$. The supernatants were transferred to fresh tubes and referred to as cytoplasmic extracts.

Colony formation assay. HCT-15P and HCT-15R cells were seeded in a 6-well plate, with 500 cells/well. The cells were treated with either different concentration of GA or $0.1 \%$ DMSO (vehicle control) and cultured in an atmosphere of 5\% $\mathrm{CO}_{2}$ at $37^{\circ} \mathrm{C}$ for the indicated times. Medium was changed 
A
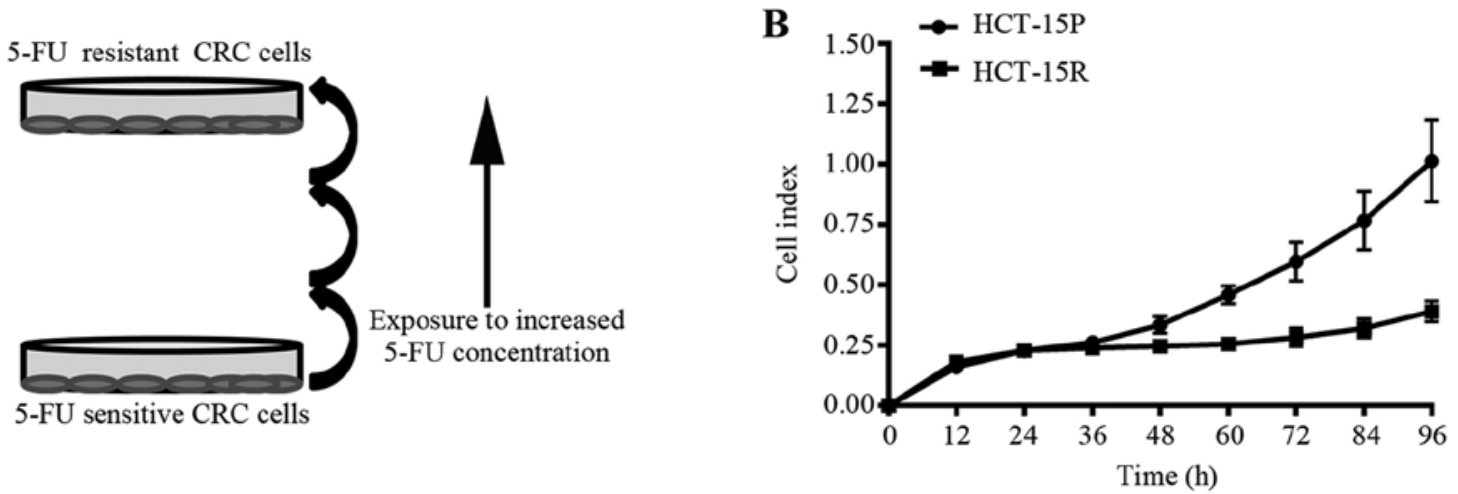

D
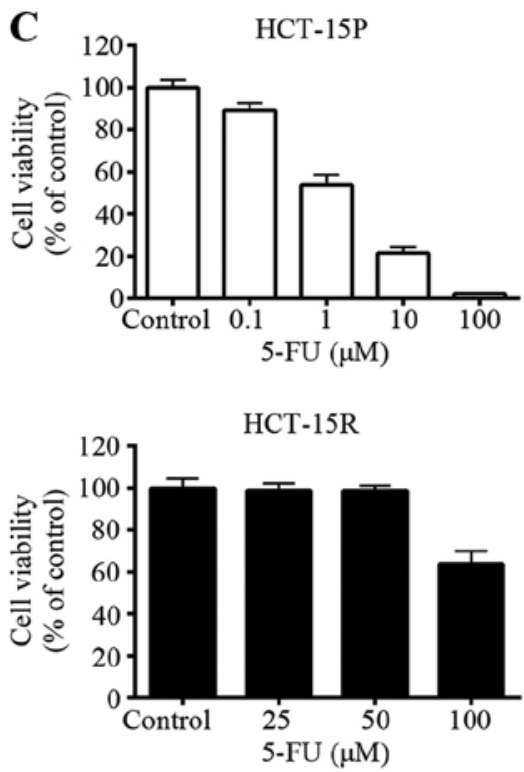

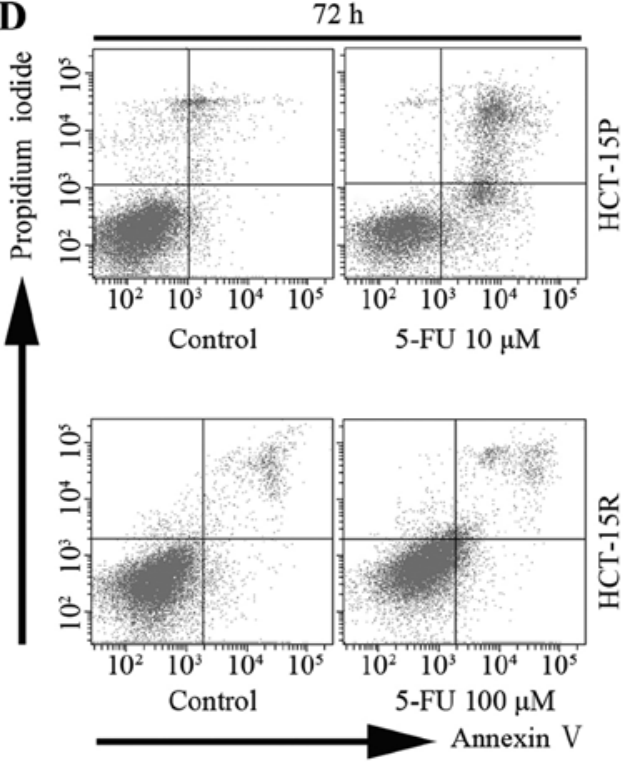

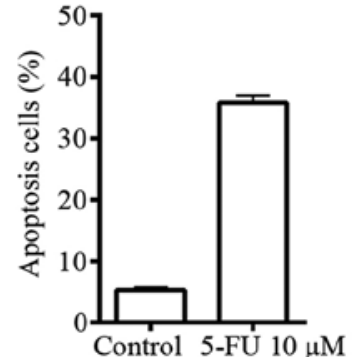

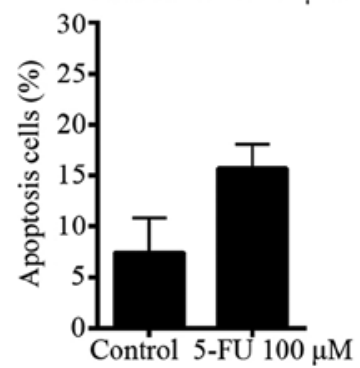

Figure 1. Characteristics of 5-FU resistant CRC cells. (A) Scheme used to generate 5-FU resistant cells. (B) Growth rate comparison in parental cells (HCT-15P) and 5-FU resistant cells (HCT-15R). The proliferation of both cells was measured by real-time cell analysis (RTCA). (C) The 5-FU sensitivity assay in HCT-15P and HCT-15R cells. Viability of HCT-15P and HCT-15R cells after treated with 5-FU at indicated concentrations was measured by MTS assay. (D) The apoptosis effects of 5-FU in both cells. Apoptotic cells stained with Annexin V-FITC and PI were analyzed by flow cytometry. The percentage of cell apoptosis was summarized (right), mean \pm SD $(n=3)$.

every 3 days. The cells were washed with PBS and fixed in ice-cold methanol for $5 \mathrm{~min}$, and stained with crystal violet. Images of the colonies were obtained using an Epson scanner. Each treatment was evaluated in triplicates, and representative images are shown.

Statistical analysis. All experiments were performed at least 3 times, and results are expressed as mean \pm SD where applicable. Statistical analysis was performed by one-way analysis of variance followed by Tukey's test by GraphPad Prism software (San Diego, CA, USA). P<0.05 was considered statistically significant.

\section{Results}

Establishment and characterization of the 5-FU resistant CRC cells. To explore the anticancer effect of GA on 5-FU resistant cells, the CRC cell line HCT-15 was subjected to successively increasing concentrations of 5-FU (Fig. 1A) to establish 5-FU resistant cells (HCT-15R). HCT-15R cells in culture grew more slowly than drug-sensitive parental cells (HCT-15P) as shown in Fig. 1B. In order to identify the establishment of resistance to
5-FU, the sensitivities of the HCT-15P cells and the HCT-15R cells were compared. Cell viability was measured using the MTS assay. HCT-15R cells were not as sensitive as HCT-15P to 5-FU (Fig. 1C). Additionally, the anti-apoptotic activity was measured using flow cytometry with Annexin V-FITC/PI dual staining (Fig. 1D). After being treated with different concentrations of 5-FU (HCT-15P cells were treated with $10 \mu \mathrm{M}$ 5-FU while HCT-15R cells were treated with $100 \mu \mathrm{M})$, there was increased apoptosis in both cell lines; however, HCT-15R cells were treated with 10 times the concentration of 5-FU and the apoptotic rate of HCT-15R was much lower than that of HCT-15P, indicating that HCT-15R showed more resistance to 5-FU.

GA effects on the proliferation and cell cycle arrest of both 5-FU sensitive and resistant cells. To investigate the anticancer effect of GA on both 5-FU sensitive and resistant CRC cells, we performed a colony formation assay. As shown in Fig. 2A, treatment of HCT-15P and HCT-15R cells with GA at concentrations from 0.0625 to $0.5 \mu \mathrm{M}$ for 7 days and 9 days, respectively, resulted in fewer and smaller colonies than those of the control group. In addition, flow cytometry analysis was 
$\mathbf{A}$

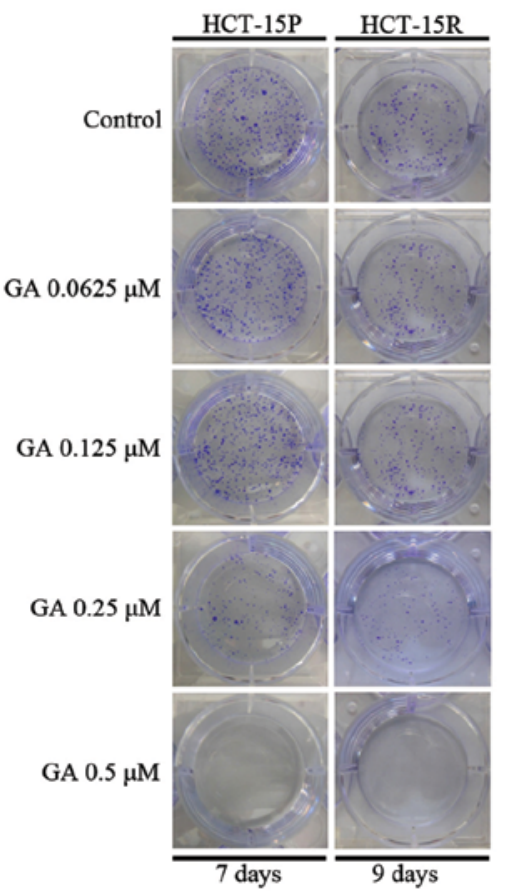

C

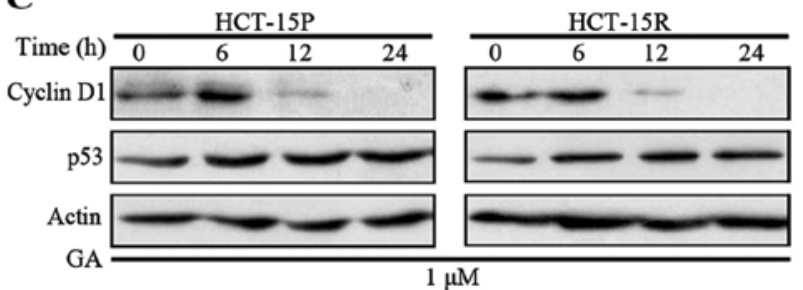

B


Figure 2. Gambogic acid (GA) inhibits cell proliferation in HCT-15P and HCT-15R cells. (A) GA inhibits the colony formation of HCT-15P and HCT-15R cells. Cells were treated with indicated concentrations $(0.0625-0.5 \mu \mathrm{M})$ of GA for 7 or 9 days. The cells were washed, fixed and stained with crystal violet. Representative images are shown. (B) The cell cycle distribution of HCT-15P and HCT-15R cells treated with GA. Cells were treated with or without GA at $1 \mu \mathrm{M}$ for indicated time (12-24 h) and stained with propidium iodide for DNA contents, followed by flow cytometric analysis. (C) The effect of GA on cell cycle related proteins. After treated with or without GA at $1 \mu \mathrm{M}$ for indicated time $(6-24 \mathrm{~h})$, cells were collected. The expression levels of cyclin D1 and p53 were detected by western blot analysis. Actin was used as loading control.

used to determine the effect of GA on cell cycle distribution. After exposing CRC cells to $2 \mu \mathrm{M}$ GA, cells were fixed, stained and analyzed by flow cytometry. As shown in Fig. 2B, there was accumulation of cells at the $\mathrm{G}_{1}$ phase, which meant GA caused $\mathrm{G}_{1}$ arrest in CRC cells. To further explore the mechanism of GA-induced cell cycle arrest, we investigated the expression of cyclin D1 and 553 proteins, which control the $\mathrm{G}_{1}$ checkpoint $(12,13)$. The results presented in Fig. $2 \mathrm{C}$ demonstrate that the addition of GA decreased the level of cyclin D1 proteins and increased the level of $\mathrm{p} 53$, which also confirmed that $\mathrm{GA}$ caused $\mathrm{G}_{1}$ arrest in CRC cells.

GA-induced apoptosis is associated with caspase activation in both HCT-15P and HCT-15R cells. Since deregulated proliferation and inhibition of apoptosis lie at the heart of all tumor development, they are the targets for therapeutic intervention in all cancers (14). We examined the ability of GA to induce cell death in HCT-15P and HCT-15R cell lines. Initially, the effect of GA on cell viability was measured by MTS assay. As shown in Fig. 3A, GA decreased the cell viability in a dose-dependent manner, with $\mathrm{IC}_{50}$ values of 1.08 and $0.87 \mu \mathrm{M}$, respectively. Additionally, the capacity of GA to induce cell apoptosis was measured by flow cytometry with Annexin V-FITC/PI dual staining. The proportion of apoptotic cells, in a time-dependent manner, increased in both HCT-15P and HCT-15R cells (Fig. 3B). To further verify the induction of apoptosis, apoptosis-associated proteins were measured by western blot assay. As shown in Fig. 3C, GA induced the cleavage of PARP (an indicator of apoptosis) in both dose- and time-dependent manner in both cell lines. Consistently, the levels of the precursor forms of caspases-3, -8 and -9 were decreased and that of the active forms of caspases- $3,-8$ and -9 were increased after GA treatment, matching the pattern of PARP cleavage, demonstrating that GA triggers CRC cell apoptosis via caspase activation.

GA-induced apoptosis is associated with the loss of mitochondrial membrane potential (MMP) and decreased expression of anti-apoptotic proteins in HCT-15P and HCT-15R cells. Mitochondria are well known to have a central role in apoptosis, which is involved in a variety of key invents, including loss of MMP, mitochondrial swelling and release of mitochondria proteins such as cytochrome $c$ and AIF from mitochondria to cytosol and/or the nucleus, which are recognized as 
A

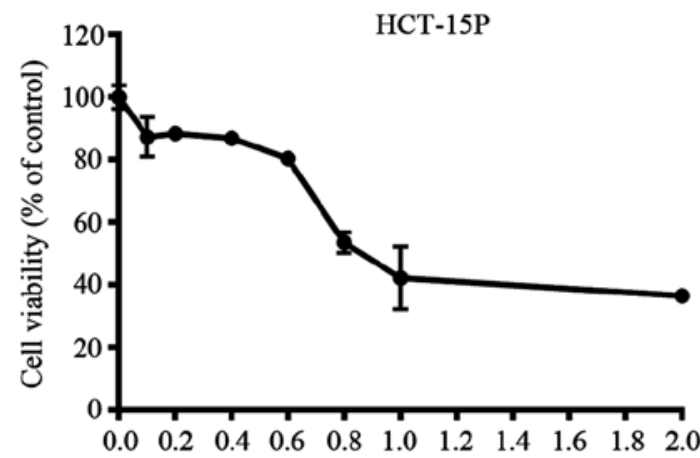

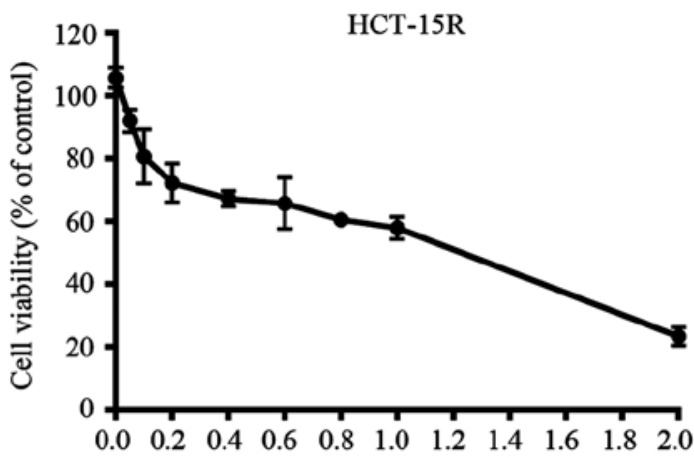

Annexin V
C

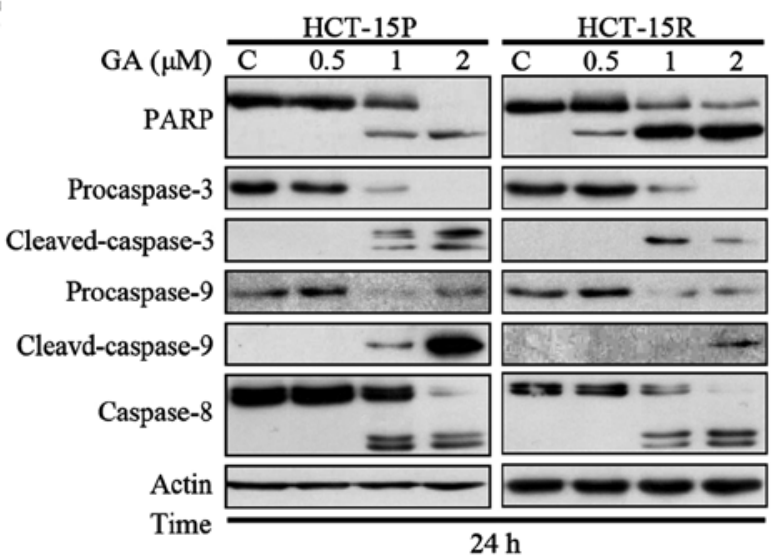

$24 \mathrm{~h}$

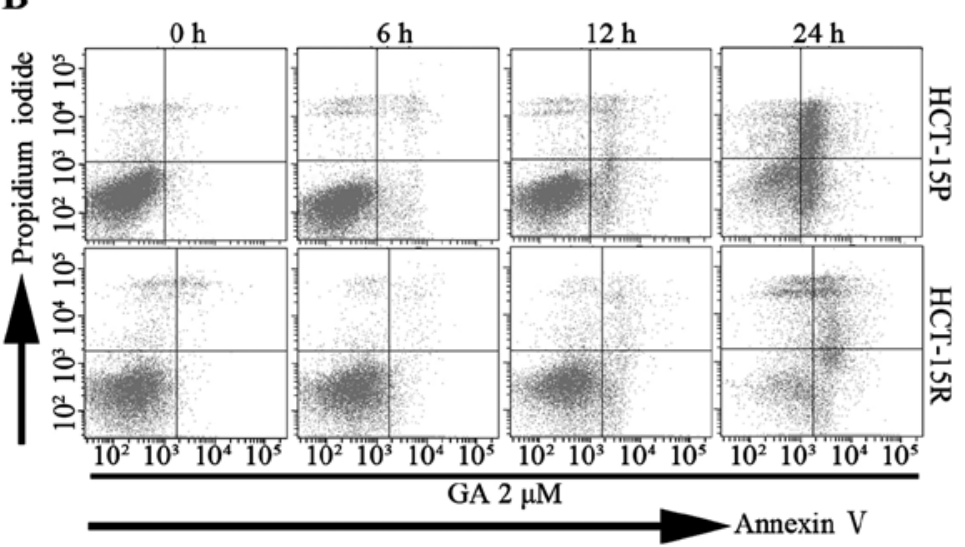

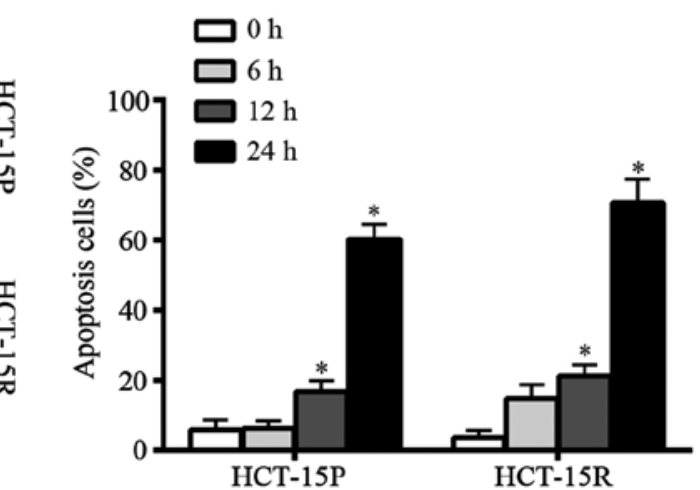

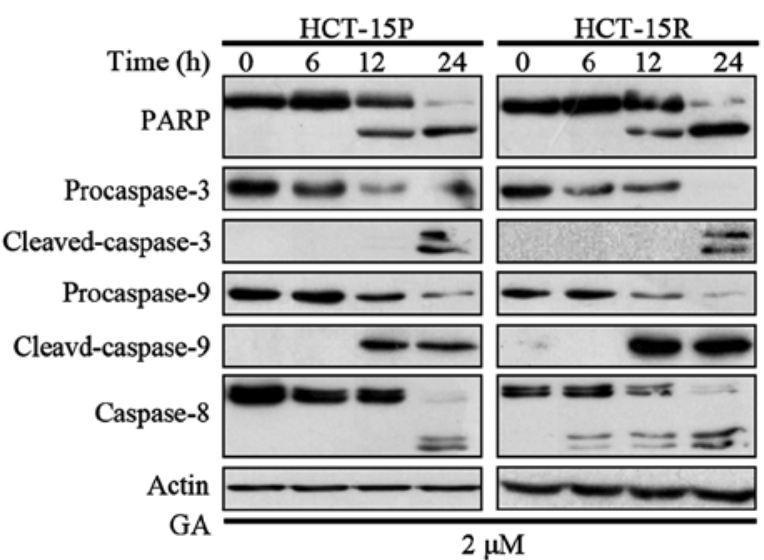

Figure 3. GA induces caspase activation in both HCT-15P and HCT-15R cells. (A) The effect of cell viability on both cell types. Viability of HCT-15P and HCT-15R cells after GA was measured by MTS assay. Cells were exposed to GA in various concentrations $(0.2-2 \mu \mathrm{M})$ for $48 \mathrm{~h}$, and then were subjected to MTS assay, mean $\pm \mathrm{SD}(\mathrm{n}=3)$. (B) The apoptosis effects of GA on both cells. Cells were treated with GA at $2 \mu \mathrm{M}$ for indicated time (6-24 h), and cell apoptosis was detected with Annexin V-FITC/PI dual staining by flow cytometry. Representative images are shown, mean \pm SD $(n=3)$. The percentage of cell apoptosis was summarized (right). ${ }^{*} \mathrm{P}<0.05$. (C) The effect of GA on caspase-related protein. Cells were treated with indicated concentrations $(0.5-2 \mu \mathrm{M})$ of GA for $24 \mathrm{~h}$ or $2 \mu \mathrm{M}$ GA for 6-24 h. PARP, procaspase-3, cleaved-caspased-3, procaspase-9, cleaved-caspase- 9 and caspase- 8 expressions were analyzed by western blot analysis. Actin was used as loading control.

indicators of the early stage of apoptosis (15). Since loss of MMP is a crucial step and subsequently triggers the release of mitochondria proteins. First, we measured the loss of MMP in GA treatment CRC cells. As shown in Fig. 4A, Both HCT-15P and HCT-15R cells treated with $2 \mu \mathrm{M}$ GA exhibited an increased green fluorescence signal and a decreased red fluorescence signal in a time-dependent manner. The percentage for loss of MMP increased to 65.37 and $69.57 \%$ in HCT-15P and HCT-15R cells, respectively, with GA in $24 \mathrm{~h}$ (Fig. 4A). Subsequently, the levels of cytosolic cytochrome $c$ and AIF were detected by western blot assay. As shown in Fig. 4B, after GA treatment, the levels of mitochondrial cytochrome $c$ and AIF increased in a time-dependent manner in both cell lines. The release of cytochrome $c$ and other apoptotic proteins from mitochondria are known to be regulated by the Bcl-2 family of proteins (16). Therefore, the expression of Bcl-2, Bcl-xl and other anti-apoptotic proteins were measured. As shown in Fig. 4C, GA decreased the level of anti-apoptotic proteins Bcl-2, Bcl-xl, Mcl-1, XIAP and survivin in both HCT-15P and HCT-15R cells in a dose- and time-dependent manner. These results demonstrated that GA-induced apoptosis is associated with loss of MMP and 
A
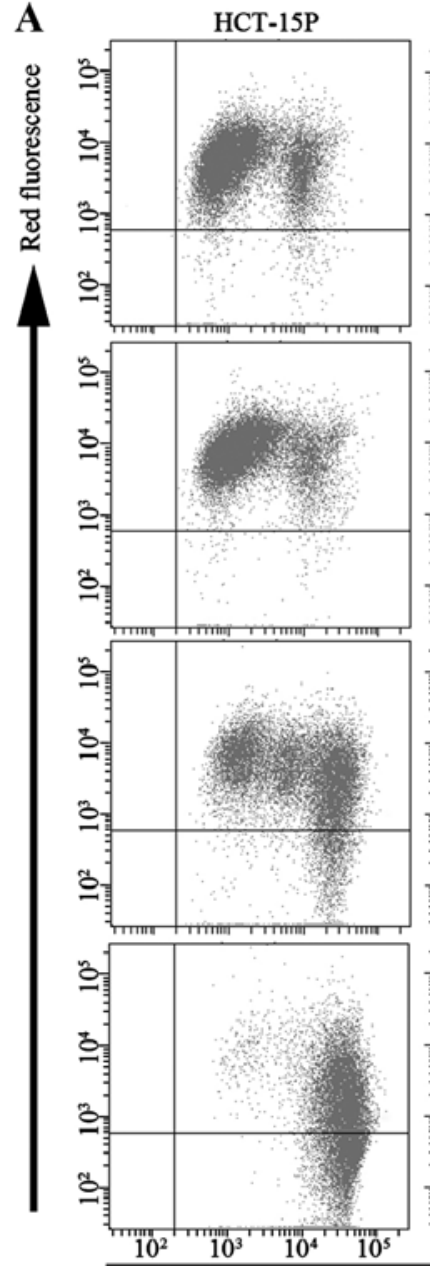

GA $2 \mu \mathrm{M}$

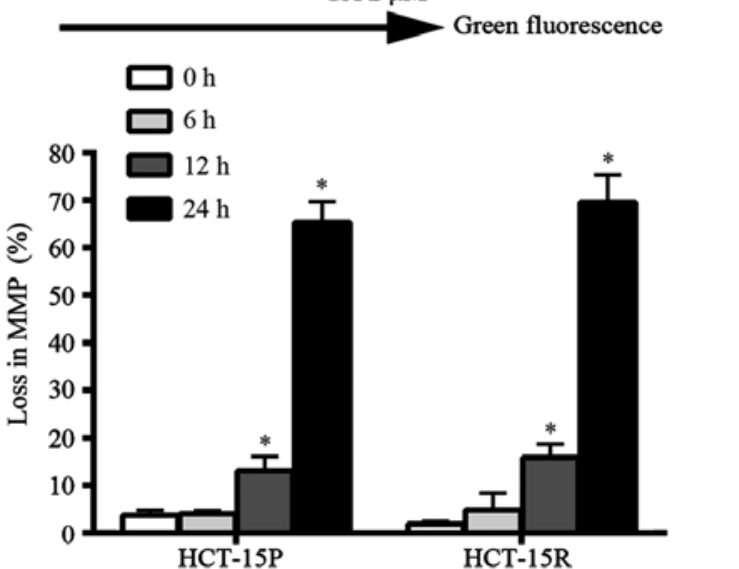

$12 \mathrm{~h}$

$24 \mathrm{~h}$
B



C
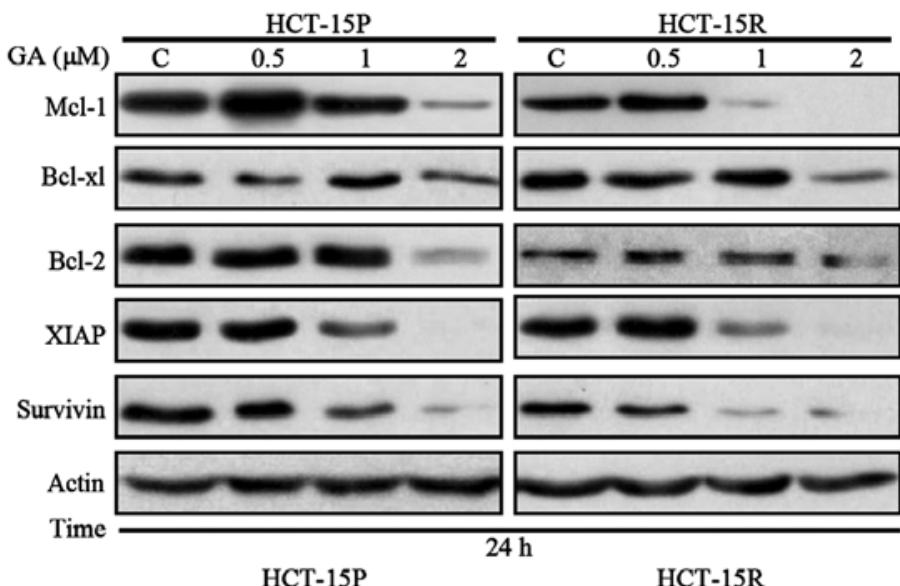

Time (h) \begin{tabular}{lllll}
\cline { 2 - 4 } & 0 & 3 & 6 & 12 \\
\cline { 2 - 5 } & & & &
\end{tabular}

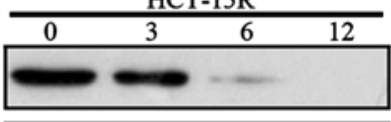

Mcl-1

Bcl-x
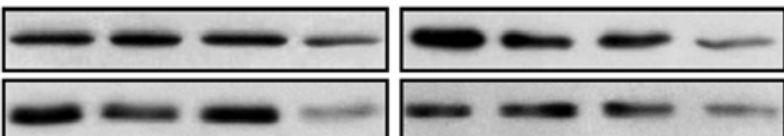

Bcl-2
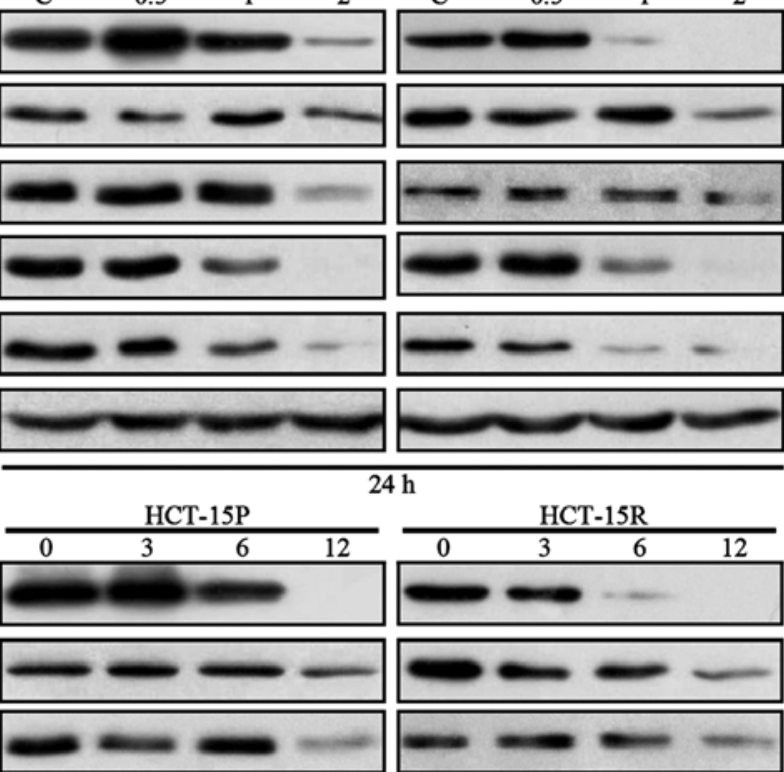

XIAP
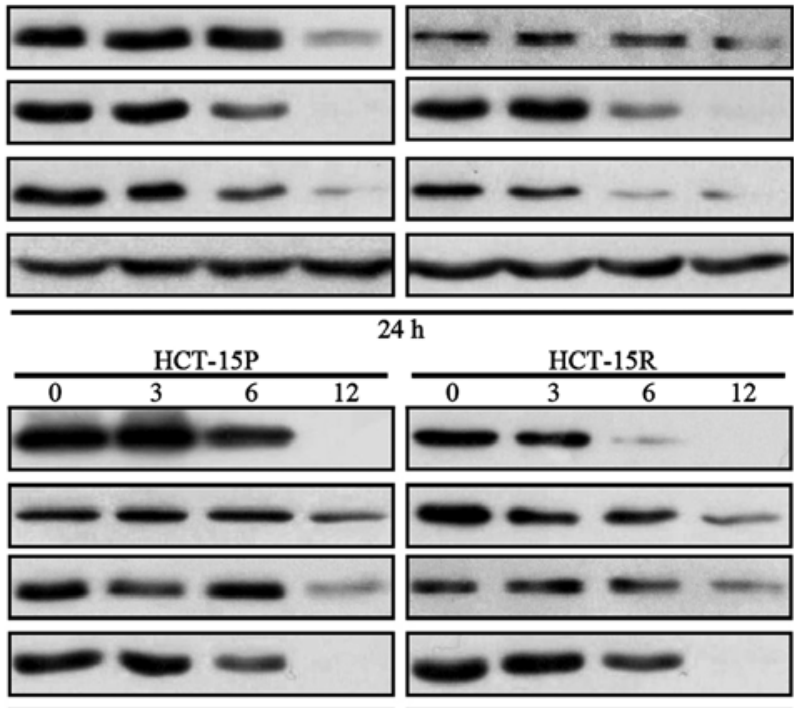

Survivin
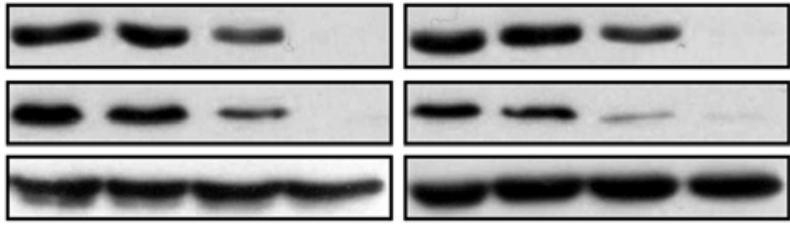

Actin

GA



Figure 4. GA disrupts mitochondrial membrane potential and decreases expression of anti-apoptotic proteins in HCT-15P and HCT-15R cells. (A) GA induces disruption of mitochondrial membrane potential (MMP). Cells were treated with $2 \mu \mathrm{M}$ GA for 6 , 12 and 24 h, and the loss of MMP was detected by JC-1 with flow cytometry, mean $\pm \mathrm{SD}(\mathrm{n}=3)$. ${ }^{*} \mathrm{P}<0.05$. (B) GA induces AIF and cytochrome $c$ release. Cells were exposed to GA at $2 \mu \mathrm{M}$ for 3,6 and 12 h, then cell cytoplasm was extracted and the released AIF and cytochrome $c$ were detected by western blot analysis. (C) GA decreases the expression of anti-apoptotic proteins in both cell types. Cells were dose- and time-dependently treated with GA. The anti-apoptotic proteins Mcl-1, Bcl-xl, Bcl-2, XIAP and survivin were analyzed by western blot analysis. Actin was used as loading control.

decreasing of anti-apoptotic proteins in both HCT-15P and HCT-15R cells.

GA-induced apoptosis is associated with activation of JNK signaling pathway in HCT-15P and HCT-15R cells. JNK activation can lead to cytotoxic effect in cancer cells. Therefore, we examined the effect of GA on the expression of this signaling pathway. The level of phosphorylated JNK (p-JNK) and total JNK was examined by western blot assay. As shown in Fig. 5, GA treatment of HCT-15P and HCT-15R cells resulted in activation of JNK, in a dose- and time-dependent manner. In addition, c-Jun expression level was elevated in parallel with increased c-Jun phosphorylation in GA treated HCT-15P and HCT-15R cells. To identify whether the JNK signaling 

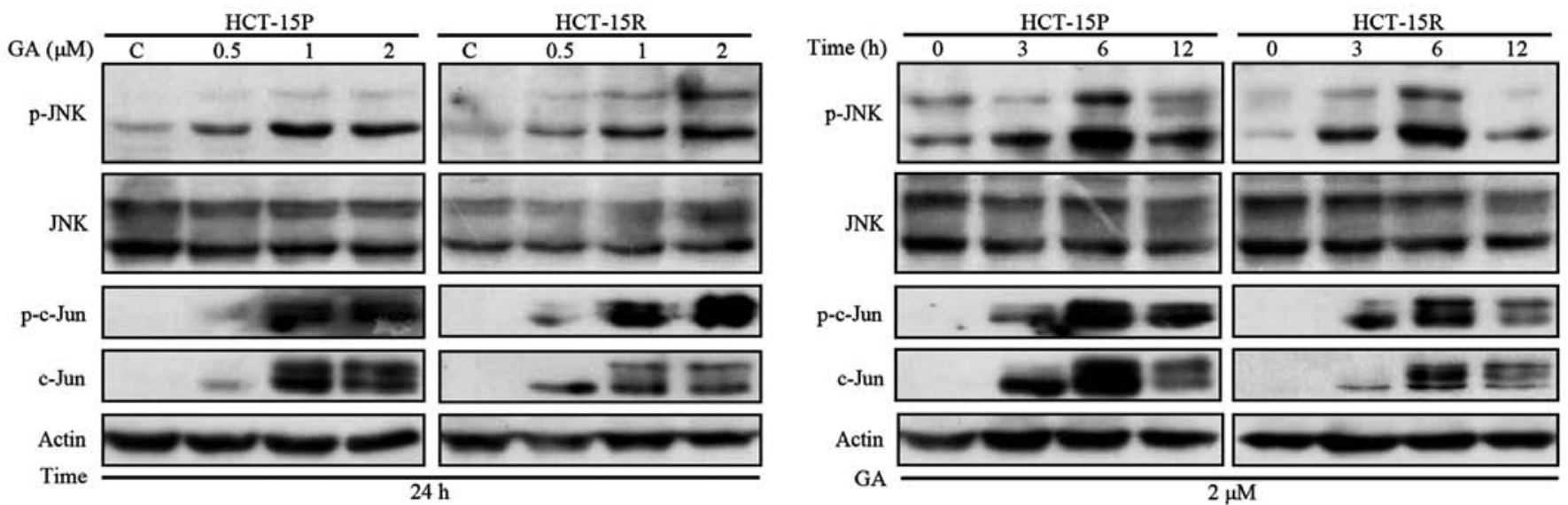

Figure 5. GA activates the JNK signaling pathways in HCT-15P and HCT-15R cells. Cells were treated with GA dose- and time-dependently, and cell lysates were analyzed by western blot analysis with antibodies against phosphorylation-JNK (p-JNK), total JNK, phosphorylation-c-Jun (p-c-Jun) and total c-Jun. Actin was used as loading control.

A

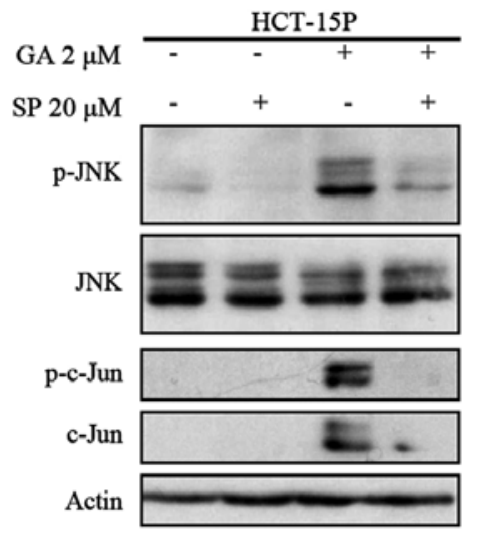

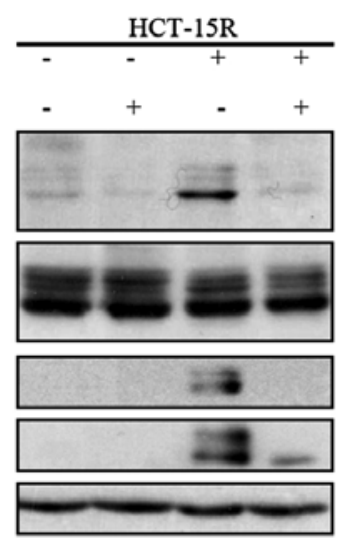

C

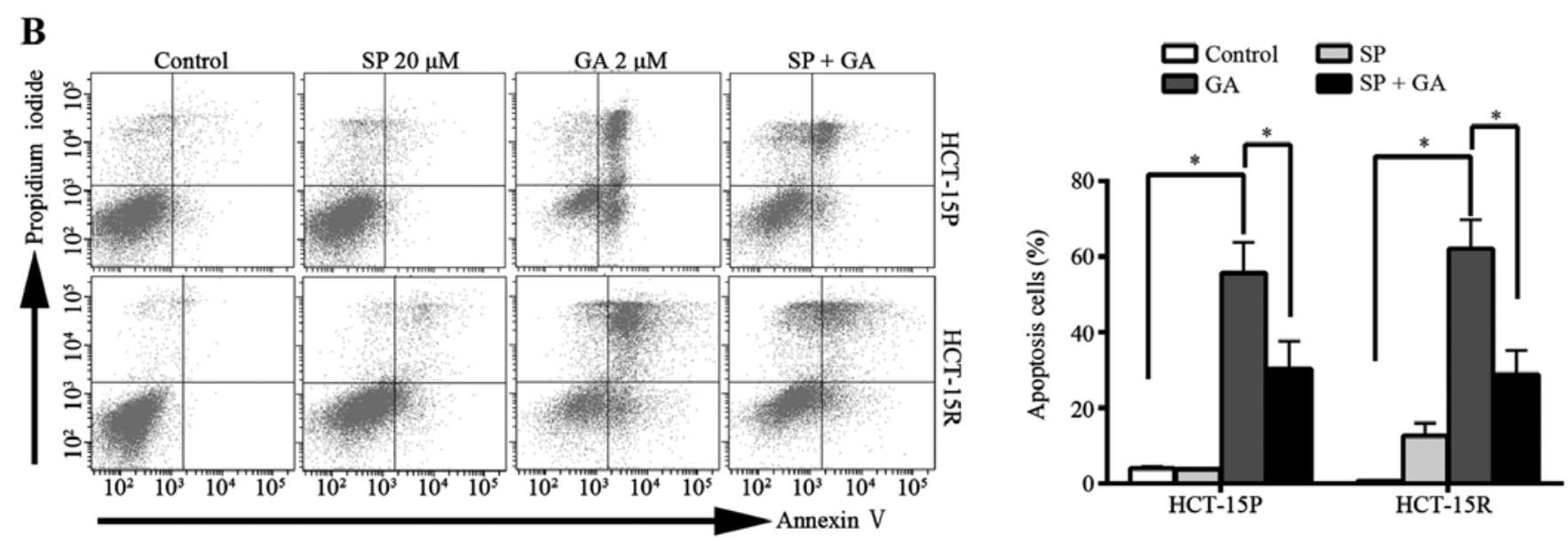

Figure 6. Activation of JNK is crucial for GA induced apoptosis in both HCT-15P and HCT-15R cells. Cells were pretreated with $20 \mu \mathrm{M} \mathrm{SP600125} \mathrm{for} 2 \mathrm{~h}$ before treatment with $2 \mu \mathrm{M}$ GA for another $24 \mathrm{~h}$. (A) Activation of JNK and its downstream targets was analyzed by western blot assay. Actin was used as loading control. (B) The percentage of cells undergoing apoptosis was determined by flow cytometry following Annexin V-FITC/PI dual staining. The percentage of cell apoptosis was summarized (right), mean $\pm \mathrm{SD}(\mathrm{n}=3)$. ${ }^{*} \mathrm{P}<0.05$. (C) The apoptosis associated protein PARP was detected by western blot analysis. Actin was used as loading control.

pathway was involved in GA-mediated apoptosis, SP600125, a general inhibitor of JNK, was pre-treated with GA in HCT-15P and HCT-15R cells and cell apoptosis was then determined using flow cytometry with Annexin V-FITC/PI dual staining. The cleavage of PARP also was detected by western blot assay. Pre-treatment with JNK inhibitor SP600125 completely blocked GA-induced phosphorylation of JNK and c-Jun (Fig. 6A). As shown in Fig. 6B, SP600125 pretreatment rescued the GA-induced apoptosis. The percentage of apoptotic cells decreased from 55.63 and 62.13 to 30.27 and $28.86 \%$ in HCT-15P and HCT-15R cells, respectively. Western blot assay showed that SP600125 inhibited the cleavage of PARP induced 
by GA (Fig. 6C), further confirming SP600125 can rescue the GA-induced apoptosis. Together, these results demonstrated that GA induced both HCT-15P and HCT-15R cell apoptosis via JNK activation.

\section{Discussion}

$\mathrm{CRC}$ is one of the most prevalent malignancies with high morbidity and mortality worldwide. Metastatic colorectal cancer (mCRC) at diagnosis is found in $25 \%$ of patients with colorectal cancer and almost $50 \%$ of patients will develop metastasis (17). For decades, the only treatment option available for mCRC patients was 5-FU. In recent years, due to new approaches in the treatment of mCRC, involving chemotherapy regimens including bevacizumab, cetuximab or panitumumab, response rates and overall survival has significantly improved (18). However, 5-FU is still widely used in the treatment of various types of cancer, including colorectal (19), breast (20) and pancreatic cancer (21). The mechanism of 5-FU resistance still remains largely unknown despite extensive investigations. Great efforts have been made to develop novel chemotherapeutic strategies for mCRC patients and to overcome chemotherapy resistance. Multiple factors could be causing chemotherapy resistance such as the alteration of drug transport, enhancement of the drug detoxification system, modification of DNA damage tolerance mechanisms and disruption of apoptotic cell death pathways. Among them, inhibition of apoptosis is considered as the most important factor of 5-FU resistance (22).

GA, a candidate drug, has been approved by CFDA for phase II clinical trial in solid tumor therapy (6). Previous studies showed that GA could induce apoptosis in a broad range of human cancer cells $(3,8,23-25)$. Thus, it is possible that GA could overcome 5-FU resistant in CRC by promoting apoptosis. To explore the potential capacity of GA to overcome 5-FU resistance in CRC cells, we established the 5-FU resistant colorectal cancer cell line HCT-15R, displaying stable biological characteristics and resistance to 5-FU. We reasoned that GA has an antitumor effect on 5-FU sensitive and 5-FU resistant cells. To test this hypothesis, we performed a series of cytotoxicity experiments on both 5-FU sensitive and 5-FU resistant cells. Our results demonstrated that $\mathrm{GA}$, inhibited cell proliferation and caused $\mathrm{G}_{1}$ arrest in both 5-FU sensitive and 5-FU resistant cells. Additionally, it induced apoptosis in both 5-FU sensitive and resistant CRC cells. Western blot analyses showed that GA induced caspase-dependent apoptosis. The above data demonstrate that GA might become an ideal agent for overcoming 5-FU resistance.

GA was reported to induce apoptosis through both death receptor apoptotic pathway and mitochondrial apoptotic pathway in hepatocellular carcinoma cells (26). On the other hand, GA was reported to induce apoptosis via a mitochondrial pathway in breast cancer cells (8) and mantle cell lymphoma cells (27). Conversely, other reports have shown the viability of an inhibition effect of GA via mitochondriaindependent apoptotic pathway on osteosarcoma cells (28). In the present study, we found that GA triggered the loss of MMP and subsequently released cytochrome $c$ and AIF to the cytosol. Cytochrome $c$ releasing from mitochondria can acti- vate caspase-9, which in turn activates executioner caspase-3 via cleavage induction, and PARP, an important substrate of caspase- 3 was cleaved. Thus our results suggest that GA could, at least partially, cause apoptosis through a mitochondrial pathway in both 5-FU sensitive and 5-FU resistant cells.

MAPKs families play an important role in complex cellular programs such as proliferation, differentiation, development, transformation and apoptosis (28). In mammalian cells, the MAPK family includes ERKs (classical MAPK), JNKs and p38 MAPKs. Usually, ERKs are activated by mitogens (29). In addition, JNKs and p38 MAPKs are most potently activated by cytokines, a variety of chemical and radiant stress and in response to cellular stress (30-32). GA was reported to induce cancer cell apoptosis through p38 MAPK activation in human breast cancer MCF-7 cells (33), Doxorubicin-resistant MCF-7/ADR cells (34) and in hepatocellular carcinoma SMMC-7721 cells (25). Evidence that JNK signaling to c-Jun contributes to stress-induced cell apoptosis have come from studies in neuron cells (32). The activation of JNK pathway impairs survival of colorectal cancer cells by promoting cell apoptosis (35). However, whether or not the JNK signaling pathway plays similar roles in GA-mediated apoptosis in colorectal cancer cells, especially in drug resistant colorectal cancer cells, is still unclear. Therefore, we investigated JNK signaling pathway after GA treatment. As the activation of JNK requires phosphorylation (p-JNK), it was observed that treatment with GA markedly promoted the expression of p-JNK. Several studies indicated that c-Jun is a specific target of JNK (32). On one hand, a variety of cellular stresses such as oxidative stress can strongly activate the JNKs, which inhibit c-Jun ubiquitination and promote the c-Jun activation through c-Jun phosphorylation. On the other hand, because the transcription factor c-Jun can regulate itself, expression via binding to its own promoter as a c-Jun/ATF-2 dimer, specific phosphorylation of c-Jun by JNK could lead to increased expression of c-Jun (36). Consistently, the active form of c-Jun (p-c-Jun) and total c-Jun protein were remarkably increased dose- and time-dependently. Furthermore, our results also showed that the inhibition with SP600125, a JNK inhibitor, partially inhibited apoptosis induced by GA, suggesting the involvement of JNK on the GA effect. Downregulation of JNK activation by SP600125, a JNK inhibitor, decreased GA-induced apoptosis, indicating that GA required the activation of JNK signaling pathways in both 5-FU sensitive and resistant cells in order to trigger the apoptotic pathway. Several studies in other cancers, such as cervical (23), breast cancer (33) and hepatic carcinoma (25), reported that inhibition of either JNK or p38 pathway led to inhibition of GA-induced apoptosis. Our results, together with these reports, highlight the important role of JNK signaling pathway in GA-induced apoptosis.

In conclusion, our results demonstrate that GA can directly inhibit proliferation and induce apoptosis in both 5-FU sensitive and 5-FU-resistant colorectal cancer cells and induce apoptosis via activating JNK signaling pathway. Therefore, data presented here suggest an alternative strategy to overcome 5-FU resistance in CRC, highlighting the importance of targeting JNK signaling pathways in anticancer strategies and their modulation by GA in CRC cells. Furthermore, we show the potential of GA to be used in CRC chemotherapy. 


\section{Acknowledgements}

This study was supported by the Guangdong Provincial Department of Science and Technology (2012B050500004; 2011B090400559); the Guangdong Innovative Research Team Program(2009010058); theScienceandInformation Technology Bureau of Guangzhou, Guangdong (2011J5200009); the Overseas Excellent Professor Project, Ministry of Education, China; and the Japan Ministry of Education, Culture, Sports, Science and Technology (MEXT) for Program of Japan Initiative for Global Research Network on Infectious Diseases (J-GRID).

\section{References}

1. Akasaka T, Tsujii M, Kondo J, Hayashi Y, Ying J, Lu Y, Kato M, Yamada T, Yamamoto S, Inoue T, et al: 5-FU resistance abrogates the amplified cytotoxic effects induced by inhibiting checkpoint kinase 1 in p53-mutated colon cancer cells. Int J Oncol 46: 63-70, 2015.

2. Qi Q, You Q, Gu H, Zhao L, Liu W, Lu N and Guo Q: Studies on the toxicity of gambogic acid in rats. J Ethnopharmacol 117 433-438, 2008.

3. Shi X, Chen X, Li X, Lan X, Zhao C, Liu S, Huang H, Liu N, Liao S, Song W, et al: Gambogic acid induces apoptosis in imatinib-resistant chronic myeloid leukemia cells via inducing proteasome inhibition and caspase-dependent Bcr-Abl downregulation. Clin Cancer Res 20: 151-163, 2014.

4. Chuah LO, Yeap SK, Ho WY, Beh BK and Alitheen NB: In vitro and in vivo toxicity of garcinia or hydroxycitric Acid: A review. Evid Based Complement Alternat Med 2012: 197920, 2012.

5. Márquez F, Babio N, Bulló M and Salas-Salvadó J: Evaluation of the safety and efficacy of hydroxycitric acid or Garcinia cambogia extracts in humans. Crit Rev Food Sci Nutr 52: 585-594, 2012

6. Chi Y, Zhan XK, Yu H, Xie GR, Wang ZZ, Xiao W, Wang YG, Xiong FX, Hu JF, Yang L, et al: An open-labeled, randomized, multicenter phase IIa study of gambogic acid injection for advanced malignant tumors. Chin Med J (Engl) 126: 1642-1646, 2013.

7. Wang LH, Yang JY, Yang SN, Li Y, Ping GF, Hou Y, Cui W, Wang ZZ, Xiao W and Wu CF: Suppression of NF- $\mathrm{BB}$ signaling and P-glycoprotein function by gambogic acid synergistically potentiates adriamycin-induced apoptosis in lung cancer. Curr Cancer Drug Targets 14: 91-103, 2014.

8. Li C, Qi Q, Lu N, Dai Q, Li F, Wang X, You Q and Guo Q: Gambogic acid promotes apoptosis and resistance to metastatic potential in MDA-MB-231 human breast carcinoma cells. Biochem Cell Biol 90: 718-730, 2012.

9. Li X, Liu S, Huang H, Liu N, Zhao C, Liao S, Yang C, Liu Y, Zhao C, Li S, et al: Gambogic acid is a tissue-specific proteasome inhibitor in vitro and in vivo. Cell Rep 3: 211-222, 2013.

10. Yi T, Yi Z, Cho SG, Luo J, Pandey MK, Aggarwal BB and Liu M: Gambogic acid inhibits angiogenesis and prostate tumor growth by suppressing vascular endothelial growth factor receptor 2 signaling. Cancer Res 68: 1843-1850, 2008.

11. Song M, Chen D, Lu B, Wang C, Zhang J, Huang L, Wang X, Timmons CL, Hu J, Liu B, et al: PTEN loss increases PD-L1 protein expression and affects the correlation between PD-L1 expression and clinical parameters in colorectal cancer. PLoS One 8: e65821, 2013.

12. Shapiro GI and Harper JW: Anticancer drug targets: Cell cycle and checkpoint control. J Clin Invest 104: 1645-1653, 1999.

13. Pines J: Four-dimensional control of the cell cycle. Nat Cell Biol 1: E73-E79, 1999.

14. Evan GI and Vousden KH: Proliferation, cell cycle and apoptosis in cancer. Nature 411: 342-348, 2001.

15. Wang X: The expanding role of mitochondria in apoptosis. Genes Dev 15: 2922-2933, 2001.
16. Tsujimoto Y: Role of Bcl-2 family proteins in apoptosis: Apoptosomes or mitochondria? Genes Cells 3: 697-707, 1998

17. Brenner H, Kloor M and Pox CP: Colorectal cancer. Lancet 383 . 1490-1502, 2014

18. Temraz S, Mukherji D, Alameddine R and Shamseddine A: Methods of overcoming treatment resistance in colorectal cancer. Crit Rev Oncol Hematol 89: 217-230, 2014.

19. Gustavsson B, Carlsson G, Machover D, Petrelli N, Roth A, Schmoll HJ, Tveit KM and Gibson F: A review of the evolution of systemic chemotherapy in the management of colorectal cancer. Clin Colorectal Cancer 14: 1-10, 2015.

20. Gandhi S, Fletcher GG, Eisen A, Mates M, Freedman OC, Dent SF and Trudeau ME: Adjuvant chemotherapy for early female breast cancer: A systematic review of the evidence for the 2014 Cancer Care Ontario systemic therapy guideline. Curr Oncol 22 (Suppl 1): S82-S94, 2015.

21. Thota R, Pauff JM and Berlin JD: Treatment of metastatic pancreatic adenocarcinoma: A review. Oncology (Williston Park) 28: 70-74, 2014.

22. Johnstone RW, Ruefli AA and Lowe SW: Apoptosis: A link between cancer genetics and chemotherapy. Cell 108: 153-164, 2002.

23. Krajarng A, Imoto M, Tashiro E, Fujimaki T, Shinjo S and Watanapokasin R: Apoptosis induction associated with the ER stress response through up-regulation of JNK in HeLa cells by gambogic acid. BMC Complement Altern Med 15: 26, 2015.

24. Zhao W, Zhou SF, Zhang ZP, Xu GP, Li XB and Yan JL: Gambogic acid inhibits the growth of osteosarcoma cells in vitro by inducing apoptosis and cell cycle arrest. Oncol Rep 25: 1289-1295, 2011

25. Nie F, Zhang X, Qi Q, Yang L, Yang Y, Liu W, Lu N, Wu Z, You Q and Guo Q: Reactive oxygen species accumulation contributes to gambogic acid-induced apoptosis in human hepatoma SMMC-7721 cells. Toxicology 260: 60-67, 2009.

26. Lee PN and Ho WS: Antiproliferative activity of gambogic acid isolated from Garcinia hanburyi in Hep3B and Huh7 cancer cells. Oncol Rep 29: 1744-1750, 2013.

27. Xu J, Zhou M, Ouyang J, Wang J, Zhang Q, Xu Y, Xu Y, Zhang Q, $\mathrm{Xu} \mathrm{X}$ and Zeng $\mathrm{H}$ : Gambogic acid induces mitochondria-dependent apoptosis by modulation of $\mathrm{Bcl}-2$ and $\mathrm{Bax}$ in mantle cell lymphoma JeKo-1 cells. Chin J Cancer Res 25: 183-191, 2013.

28. Zhao W, You CC, Zhuang JP, Zu JN, Chi ZY, Xu GP and Yan JL: Viability inhibition effect of gambogic acid combined with cisplatin on osteosarcoma cells via mitochondria-independent apoptotic pathway. Mol Cell Biochem 382: 243-252, 2013.

29. Fang JY and Richardson BC: The MAPK signalling pathways and colorectal cancer. Lancet Oncol 6: 322-327, 2005.

30. Dhanasekaran DN and Reddy EP: JNK signaling in apoptosis. Oncogene 27: 6245-6251, 2008.

31. Zarubin T and Han J: Activation and signaling of the p38 MAP kinase pathway. Cell Res 15: 11-18, 2005.

32. Dunn C, Wiltshire C, MacLaren A and Gillespie DA: Molecular mechanism and biological functions of c-Jun $\mathrm{N}$-terminal kinase signalling via the c-Jun transcription factor. Cell Signal 14: 585-593, 2002.

33. Chen J, Gu HY, Lu N, Yang Y, Liu W, Qi Q, Rong JJ, Wang XT, You QD and Guo QL: Microtubule depolymerization and phosphorylation of c-Jun N-terminal kinase-1 and p38 were involved in gambogic acid induced cell cycle arrest and apoptosis in human breast carcinoma MCF-7 cells. Life Sci 83: 103-109, 2008.

34. Wang S, Wang L, Chen M and Wang Y: Gambogic acid sensitizes resistant breast cancer cells to doxorubicin through inhibiting P-glycoprotein and suppressing survivin expression. Chem Biol Interact 235: 76-84, 2015

35. Favata MF, Horiuchi KY, Manos EJ, Daulerio AJ, Stradley DA, Feeser WS, Van Dyk DE, Pitts WJ, Earl RA, Hobbs F, et al: Identification of a novel inhibitor of mitogen-activated protein kinase kinase. J Biol Chem 273: 18623-18632, 1998.

36. Raivich G, Bohatschek M, Da Costa C, Iwata O, Galiano M, Hristova M, Nateri AS, Makwana M, Riera-Sans L, Wolfer DP, et al: The AP-1 transcription factor c-Jun is required for efficient axonal regeneration. Neuron 43: 57-67, 2004. 